\title{
The Ecology of Technological Progress: How Symbiosis and Competition Affect the Growth of Technology Domains
}

\author{
Gianluca Carnabuci, University of Lugano
}

We show that the progress of technological knowledge is an inherently ecological process, wherein the growth rate of each technology domain depends on dynamics occurring in other technology domains. We identify two sources of ecological interdependence among technology domains. First, there are symbiotic interdependencies, implying that the rate of growth of one technology domain is driven by the advances made in other technology domains. Second, some technology domains compete with each other, implying that the rate at which a given technology domain advances varies inversely with the competitive pressure it receives from other technology domains. Based on all the technological knowledge patented in the United States between 1975 and 1999, we find statistical support for our argument and hypotheses.

The question of why some technology domains grow faster than others has drawn the attention of both sociologists and economists (Carnabuci 2006). As a result, several factors have been identified that bear on this question. Some scholars have argued that the uneven progress of technological knowledge reflects the changing needs of the market (Schmookler 1966), while others have emphasized the importance of institutional regimes (Bonaccorsi and Thoma 2007). Also, it has been argued that the growth of technological knowledge depends on "supply-push" factors, such as the ease and precision with which experimental tests can be carried out in a given technology domain (Nelson 2003), its inherent technical bounds (Girifalco 1991), and its degree of specialization (Carnabuci and Bruggeman 2009). Furthermore, the progress of technology domains has been argued to hinge on the social construction of what becomes legitimately accepted as "new" knowledge by the relevant epistemic community (Latour 1987; Knorr-Cetina 1999).

An implicit assumption common to all these explanations is that technology domains grow independently of each other. Departing from this assumption, our main contention in this paper is that technology domains evolve in an ecologically interdependent fashion. We reckon that two kinds of ecological pressure impact the growth of technology domains. First, there may be symbiosis among some technology domains. That is, the rate of growth of one technology domain may be accelerated by the advances made in other technology domains. Second, some technology domains may be in competition with each other. When this

The author thanks Jeroen P. Bruggeman for his cooperation in an earlier version of this article, Alfonso Gambardella for his useful insights and support, and the editor and anonymous reviewers for their constructive criticisms and suggestions. This research was supported by the Netherlands Organization for Scientific Research, NWO grant \#401-01-098, and by the Research Division of SDA Bocconi School of Management. Direct correspondence to Gianluca Carnabuci, Institute of Management, University of Lugano, Via Buffi 13, 6904, Lugano, Switzerland. E-mail: gianluca.carnabuci@usi.ch. 
happens, the rate at which a given technology domain advances can be expected to vary inversely with the competitive pressure it receives from other technology domains. Due to symbiosis and competition, technological progress may therefore be described as an ecological process wherein the growth rate of each technology domain depends on dynamics occurring in other technology domains.

\section{An Ecological View of Technological Progress}

Nowadays, there is widespread agreement that the generation of new technological knowledge is ultimately driven by the novel recombination of existing knowledge (e.g., Nelson and Winter 1982; Weitzman 1998; Jones 2005; Fleming 2007). As the historian Usher (1929:11) was early in recognizing, "[i]nvention finds its distinctive feature in the constructive assimilation of pre-existing elements into new syntheses, new patterns, or new configurations [...]." Reflecting the view that technological progress largely occurs within path-dependent trajectories of accumulation (Dosi 1982), received research has underscored that the bulk of knowledge recombination takes place within the boundaries of well-circumscribed technology domains. Consistent with this view, extant models and empirical studies have focused on the historical development of single technology domains (Girifalco 1991; Malerba et al. 1999).

Recently, however, a different and complementary perspective was offered by Carnabuci and Bruggeman (2009). To explain why certain technology domains grow faster than others, the authors built on the premise that a non-negligible share of the knowledge generated in any technology domain results from the recombination of knowledge developed in other technology domains. Consistent with this perspective, the authors proposed to conceptualize the growth of technological knowledge as an evolving network of knowledge recombination patterns across technology domains. Figure 1 presents a simple visual example of the authors' proposed network model (Carnabuci and Bruggeman 2009).

"Figure 1 is an example of such a network which depicts a cross-cut of the growth process of a hypothetical stock of public technological knowledge within a given time window. This stock consists of three technology domains, A, B and C, in which 30, 40 and 10 new inventions, respectively, have accumulated over the given time interval. The arcs point to the domains from which ideas are taken and are drawn in the direction of knowledge search. Knowledge flows in the opposite direction of the arrows, though, and in the literature on diffusion (Rogers 2003), arrows are in line with the flows. The inventions generated in A resulted from the recombination of inventions from A's own knowledge base 25 times, from B's 30 times, and from C's 20 times. Or, equivalently, knowledge spilled over 25 times from past to current inventions in A, 30 times from past inventions in B to current inventions in $\mathrm{A}$ and 20 times from past inventions in $\mathrm{C}$ to current inventions in $A$. During the same time interval, the inventions generated in $A$ worked as an input for inventions in B 40 times, i.e., ideas spilled over from A to B 40 times." 


\section{Figure 1. Recombination Patterns Within and Between Technology Domains}

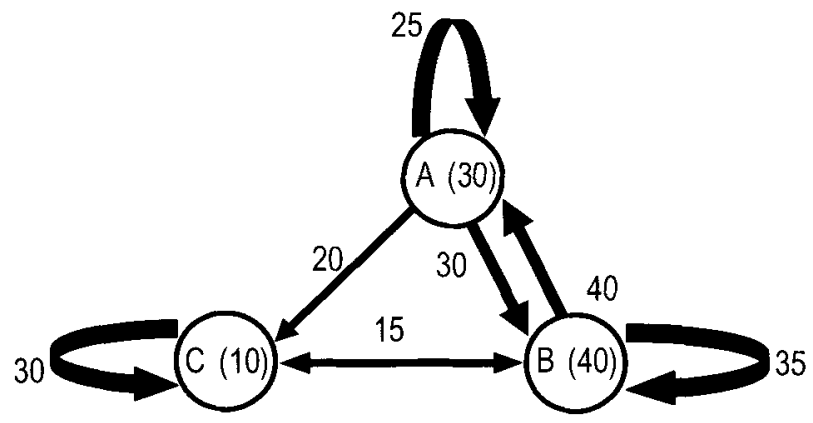

Source: Carnabuci and Bruggeman (2009)

In this article, we build on Carnabuci and Bruggeman's network conception to argue and show that technological progress is an inherently ecological process. In particular, our contention is that two kinds of ecological interdependence-symbiosis and competition-arise among technology domains due to the dynamics of knowledge recombination that underlie technological progress. ${ }^{1}$

\section{Symbiotic Interdependencies among Technology Domains}

In general terms, symbiotic interdependencies exist among two entities to the extent that they derive some kind of fit benefit from each other. ${ }^{2}$ In biology, for example, the ocellaris clownfish is known to entertain a mutually symbiotic relationship with the Ritteri sea anemones. By residing among the anemones' tentacles, the clownfish guards the anemone from anemone-eating fish, while in turn the tentacles of the anemone fence off the clownfish from its predators. Symbiotic phenomena occur outside the biological sphere as well. And indeed in sociology, the role of symbiotic interdependencies has soon been recognized as essential to many diverse social dynamics (Durkheim 1997[1893]), including social influence (Park 1936), social cohesion (Gross 1956), organizational survival (Barnett and Carroll 1987), urbanization (Hawley 1950), and state formation (Ingram and Simons 2000). Similarly, our contention is that symbiotic interdependencies may intervene in the process of technological knowledge growth, implying that the progress of one technology domain is driven by the progress of other technology domains.

While the role of symbiotic interdependencies across technology domains has not yet been systematically investigated (Pistorius and Utterback 1997), the limited available empirical evidence provides some support to this possibility. For example, in his analysis of technological change, Girifalco (1991:43) noticed that quite often "bottlenecks in one technology may call forth advances in another, 
advances in one may open up opportunities in another, or progress in one may have to await improvements in the other." Furthermore, the history of human inventions abounds with anecdotal evidence suggesting that symbiotic interactions across technologies may constitute an important and pervasive mechanism of technological progress. To name a few notable cases, the invention of the electric engine triggered new fruitful technological trajectories in the fields of refrigeration, washing and vacuum cleaning, among others. Similarly in more recent times, advances in the field of biotechnology have spawned new technological developments in a wide range of technology domains from agricultural chemicals to pharmaceutical drugs, while important technological advances in the biotechnology field itself have resulted from technologies developed in the fields of biorobotics and information technology.

Providing a general theoretical interpretation of these examples, the knowledge recombination view suggests that the growth of any given technology domain is enhanced by developments occurring in other domains, granted that some aspects of these developments are productively combined into the focal technology domain. From this perspective, the existence of symbiotic interdependencies across technology domains is neither a marginal nor an exceptional phenomenon. Rather, it is an inherent characteristic of the process of knowledge recombination through which technological knowledge advances. The argument that the process of knowledge recombination engenders interdependencies among technology domains has a straightforward and testable implication. Namely, the more heavily a technology domain depends on another as a source of knowledge recombination, the more the growth rate of the former will tend to be driven by the growth rate of the latter.

Because it captures the extent to which technology domains are dependent on each other as sources of knowledge recombination, Carnabuci and Bruggeman's network model provides a general framework through which the effect of symbiotic interdependencies across technology domains can be both formally represented and empirically tested. Let us consider again the illustration in Figure 1, wherein technology domain A recombines knowledge from both technology domain B and technology domain $\mathrm{C}$, within a given time interval $t$. In this situation, our proposed ecological perspective implies that A's growth rate will be to some extent dependent on the growth rate of both $\mathrm{B}$ and $\mathrm{C}$, because the faster $\mathrm{B}$ and $\mathrm{C}$ develop, the more abundant will be the pool of new technological knowledge that can potentially spill over to A. By the same token, the more sluggish are B's and C's growth rates, the smaller the pool of potentially symbiotic technological developments that A can benefit from. While we expect A to be symbiotically interdependent with both $B$ and $C$, however, we do not expect the strength of such interdependencies to be equal. Rather, because $\mathrm{B}$ is for $\mathrm{A}$ a more important source of knowledge recombination than is $C$, our contention is that A's growth will be more heavily affected by the rate of development of $B$ than by that of $C$. Generalizing these arguments, our first hypothesis can be expressed as follows. 
H1: The growth rate of any technology domain depends on the growth rates of the technology domains from which it recombines knowledge; furthermore, the more important is a technology domain as a source of knowledge recombination for the focal technology domain, the stronger is its impact on the focal domain's growth rate.

\section{Competitive Interdependencies Among Technology Domains}

Unlike most economic resources, technological knowledge is a non-rival good, i.e., it can be used infinitely many times without depleting (Arrow 1962; Romer 1986). Given that the input to new knowledge is existing knowledge, it is therefore often assumed that the process of knowledge recombination is immune from the ecological pressures of competition. Consistent with this view, for example, economist Martin Weitzman proposed in an influential pair of papers that knowledge growth be represented as a combinatorial process wherein new ideas can be unboundedly recombined from existing ideas (Weitzman 1996, 1998). Indeed, this view has had a substantial impact on both economic theory, leading to the research program often labeled New Growth Theory (see Jones 2005 for a critical review), and on growth and innovation policies worldwide. To the best of our knowledge, however, the assumption that the knowledge recombination process is immune from competitive interdependencies has never been tested empirically. Rather, in a study at the level of individual patents, Podolny and Stuart (1995) showed that whether a patented invention has an impact or, on the contrary, it becomes an inconsequential deadend, depends to a relevant extent on how crowded is the niche of source ideas the invention recombined. Therefore, the authors concluded that there is competition among technological ideas.

Similarly, we suspect that there may be competitive interdependencies among technology domains. We reckon that there may be two sources of competition among technology domains. First, as said, a new idea springs from the novel recombination of existing knowledge. Therefore, even if knowledge inputs could in principle be recombined infinitely many times without depleting, one should expect the value of each recombination to be directly proportional to its novelty. To the extent that technology domains draw from the same source ideas, it is likely that they reduce the novelty of each other's recombinations. Second, ultimately all inventions are the product of boundedly rational human beings, whether operating as lone inventors or, more typically, within an organization. Hence, technology domains may be in competition with each other for the limited attention of inventors (Podolny and Stuart 1995) and, relatedly, for the research and development investments made by the organizations they work for.

But what determines the structure and the intensity of competitive interdependencies among technology domains? Elaborating on a well-established notion in sociology (Podolny et al. 1996; Popielarz and Neal 2007), we propose that 
whether technology domains compete with each other depends on the degree to which they recombine knowledge from the same niche of source ideas. Our argument is as follows. The more two or more technology domains draw from a similar niche of source ideas, the more their knowledge recombinations, and therefore their newly developed ideas, are likely to be similar. As a consequence, the average portion of truly novel knowledge that each new recombination yields will decrease. Furthermore, as similarity increases and novelty decreases, both investors and inventors will tend to choose one technology and not a similar other, or they will look for more fruitful opportunities in less crowded regions of the technological landscape. This argument leads to our second hypothesis.

\section{H2: The greater the niche overlap of a technology domain, the lower its growth rate.}

To disambiguate our concepts and hypotheses, in the next section we will develop a pair of network-analytic measures capturing symbiotic and competitive interdependencies among technology domains.

\section{Formalizing the Argument}

To formally model the structure of symbiotic and competitive interdependencies among technology domains, our starting point is what Carnabuci and Bruggeman (2009:612) define a technology domain's recombinant niche, i.e., "the sub-network comprising a focal domain, the domains from which it recombines ideas (the source domains), the valued and directed ties linking the focal domain to its source domains, and the valued and directed ties linking source domains among each other."

\section{Modeling Symbiotic Interdependencies}

With regard to symbiotic interdependencies, we have argued that the more a technology domain is dependent on another technology domain as a source of knowledge recombination, the more the growth of the latter will affect the growth of the former. To formalize and, subsequently, test this idea, we follow a consolidated approach in the social network literature and use a "network autocorrelation" model (Leenders 2002). Network autocorrelation models capture the extent to which the outcome variable of a focal node in a network varies as a function of the outcome variables of its direct network contacts. In this article, we use an autocorrelation model to capture the degree to which the growth rate of a focal technology domain is impacted by the growth rates of the technology domains in its recombinant niche. Following Doreian cum suis (2004), we use the following model:

$$
s y_{i t}=\sum_{\substack{i=1 \\ i \neq j}} w_{i j t} y_{j t}
$$

where $y_{j t}$ indicates the growth rate of each technology domain, $j$, in $i$ s recombinant niche during $t, w_{i j t}$ is the impact, or weight, that each technology domain $j$ is 
expected to exert on the growth rate of $i$; and, $s y_{i t}$ is the overall impact exerted on the growth rate of domain $i$ by the domains in its recombinant niche. Because our theoretical argument is that $w_{i j t}$ varies with the relative importance of each $j$ as a source of knowledge recombination for $i$, we further assume that the larger is the proportion of knowledge that $i$ recombines from $j$, the higher is $w_{i j f^{*}}$

Let us now show how the model works by comparing two hypothetical technology domains, $i$ and $k$. Of the knowledge that technology domain $i$ recombines from other technology domains during $t$, suppose that 30 percent comes from domain $j_{i}, 20$ percent from domain $j_{2}, 40$ percent from domain $j_{3}$, and 10 percent from domain $j_{4}$. Also assume that during $t, j_{1}$ grows by 3 percent, $j_{2}$ grows by 7 percent, $j_{3}$ grows by 5 percent, and $j_{4}$ decreases by 9 percent, relative to $t_{r}$. Now consider technology domain $k$. Of the knowledge that technology domain $k$ recombines from other technology domains during $t$, let us assume that 10 percent comes from domain $j_{5}, 70$ percent from domain $j_{6}$ and 20 percent from domain $j_{3}$. Let us further assume that relative to $t_{1}, j_{5}$ decreases by 5 percent, while $j_{6}$ and $j_{5}$ grow by 30 percent and 15 percent, respectively. Under these conditions, our argument and model imply that:

$s y_{i t}=.3 \times .03+.2 \times .07+.4 \times .05-.1 \times .09=.009+.014+.02-.009=.034$

$s y_{k t}=-.1 \times .05+.7 \times .3+.2 \times .15=-.005+.21+.03=.235$

Hence in this example, our expectation is that during $t$ the growth of both $k$ and $i$ will be accelerated by the symbiotic interdependencies they entertain with other technology domains; however, this acceleration should be almost seven times as fast for $k$ as it is for $i^{3}$

\section{Modeling Competitive Interpendencies}

With regard to competitive interdependencies, our argument is that a focal technology domain is subjected to competitive pressure by other technology domains insofar as its recombinant niche overlaps with the recombinant niches of other technology domains. Accordingly, we define the dyadic niche overlap $\alpha_{i k, t}$ of a focal domain $i$ by another domain $k$ as the proportion of $i$ s recombinations that $i$ and $k$ carry out from the same source technology domains within the same time interval:

$$
\alpha_{i k, t}=\frac{\sum_{j}\left(H_{j i, t} \cap H_{j k, t}\right)}{\sum_{j} H_{j i, t}}
$$

where $\mathrm{i} \neq \mathrm{k}$ and $\mathrm{r} \neq \mathrm{j}$, and $\mathrm{t}$ is a time interval, and $\mathrm{H}$ stands for the set of recombinations carried out by the domain in the right-hand subscript, from ideas belonging to the domain in left-hand subscript.

Figure 2 presents an example. During interval $t$, domain $i$ recombined 100 times ideas belonging to domain $j_{1}, 50$ times ideas from $j_{2}$, and 60 times ideas 
from $j_{3}$. Meanwhile, domain $k$ recombined 20 times ideas from $j_{1}, 90$ times ideas from $j_{2}$, and 75 times ideas from $j_{4}$. Thus, $\alpha_{i k, t}=(20+50) / 210=.3$.

This network-analytic measure of dyadic niche overlap is continuous; it is normalized within the interval $[0,1]$; and, it takes into account units' size differences and is therefore asymmetric. ${ }^{4}$ Thus, it meets the three conditions necessary for a correct operationalization of dyadic niche overlap (Sohn 2001). The overall overlap that is niche is subjected to, $A_{i t}$, can be simply calculated as the sum of its dyadic niche overlaps,

$$
A_{i t}=\sum_{k \neq i} \alpha_{i k, t}
$$

This overall measure is similar to Podolny cum suis' (1996:666) niche crowding measure, but for the $a$-terms we do not just have to deal with "plain" niche overlaps, because in our case niches consist of pair-wise disjoint subsets. To relate a domain's competitive interdependencies to its growth rate, we then simply take the difference in the overall niche overlap of a technology domain between subsequent time periods.

\section{Empirical Data}

To test our hypotheses, we use the very same dataset used by Carnabuci and Bruggeman in their study (2009), which guarantees comparability of the results and facilitates knowledge accumulation. The data, which have been collected by the National Bureau for Economic Research and are freely and publicly accessible, comprise all patents (over two million) granted by the United States Patent and Trademark Office between 1975 and 1999, as well as all citations (over 16 million) among them. Extensive descriptive statistics and methodological discussions concerning these data are available in Hall cum suis (2001). Following Carnabuci and Bruggeman, we use these data to indicate (1. the network nodes, i.e., the technology domains; (2. their directed and valued ties, i.e. the knowledge recombination patterns within and among technology domains, and (3. the growth rate of each technology domain.

\section{Network Nodes}

The USPTO has developed an articulated system of classification (the United States Patent Classification, henceforth USPC), so that every patent can be identified by the technical content of its subject matter. In 1999, the last observation year, there were 418 domains of technological knowledge, or primary classes, and over 120,000 subclasses. Following Carnabuci and Bruggeman (2009), we used the USPC primary classes to carry out a homomorphic mapping of the acyclic network of individual patents and patent citations onto a cyclic network of technology domains and cross-domain citations. The choice of indicating technology domains through USPC primary classes is generally regarded as methodologically 
Figure 2. Niche Overlap of Two Hypothetical Technology Domains, $i$ and $k$

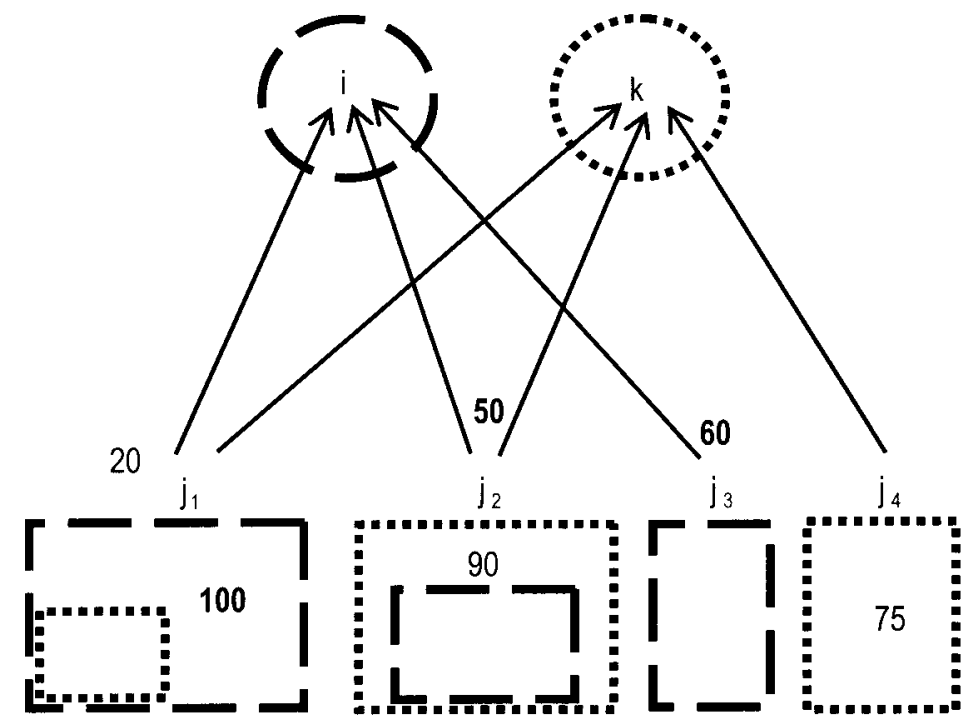

appropriate, particularly because primary classes partition patented knowledge into non-overlapping technology domains, they are more reliable and robust than other partitions, and retrospective patent reassignments are extremely rare (Henderson et al. 2005). Accordingly, USPC primary patent classes have been widely used to indicate domains of technological knowledge, providing indirect validation for this measure (see, among many others, Jaffe et al. 1993). In addition, the choice of USPC primary classes to study technological progress is theoretically grounded. Technological progress is an inherently public process, which manifests itself whenever new knowledge accumulates within the boundaries of what comes to be institutionalized as distinct technology domains. USPC primary classes provide a straightforward unit of analysis to study these domains and the accumulation process they undergo because, compared to higher or lower classifications, at this level of observation the boundaries of technology domains are both more neatly circumscribed from a strictly technical viewpoint and more salient from a socio-cognitive and institutional one (for an extensive discussion of these issues, see Carnabuci and Bruggeman 2009).

\section{Network Ties}

To model the valued and directed ties between technology domains, i.e., the cross-domain knowledge recombination patterns, we relate the USPC patent classes by patent citations. Patent citations can be obtained from the so-called prior art, a list of all previously patented inventions a focal patent draws from. By signalling which bits of disclosed knowledge a patented invention draws from 
(Jaffe et al. 1993), patent citations are indicative of the knowledge recombination underlying the newly generated invention. Since Griliches' seminal work (1979), dozens of econometric studies have exploited this property of patent citations to investigate knowledge recombination patterns (e.g., Jaffe 1986). In addition to indirect evidence, two validation studies (Jaffe et al. 1998, 2000) concluded that patent citations are a valid measure of knowledge recombination. ${ }^{5}$

\section{Domains' Growth Rate}

The more a patented invention consists of knowledge that is useful for the generation of new inventions, the more subsequent patents will cite it. Therefore, a widely established method to measure how much a patent contributed to the advancement of technological knowledge is counting the number of citations it received, also called forward citations (e.g., Griliches 1990). Indicators of knowledge output based on forward citations have received empirical validation from all studies known to us. Besides indirect evidence, their validity was confirmed by three validation studies (Albert et al. 1991; Jaffe et al. 2000; Albert et al. 2002). To measure the knowledge output of a technology domain within a given interval, we count the total number of patents generated in that technology domain, weighed by the number of citations each of these patents received, within that time interval. Then, to calculate the growth rate of a technology domain-our dependent variable-we take the percentage growth between that domain's knowledge output over subsequent time windows. This brings us to the problem of choosing the length of time intervals, which we discuss next.

\section{Network Evolution}

Based on the abovementioned modeling specifications, we constructed our knowledge recombination network. To capture the evolution of this network, we modeled each network cross-section based on all citations between patents granted within the same time window. For the length of the time window, we chose five years, following a common practice (e.g., Podolny et al. 1996). While the choice of five years is somewhat arbitrary, Carnabuci and Bruggeman (2009) devised a number of tests that show that it is an appropriate way to model the evolution of our knowledge recombination network. Further, to model the network crosssections from entirely non-overlapping data, we reduced the number of intervals to five non-overlapping networks. As a consequence, the evolving network of patent citations between technology domains in the years from 1975 to 1999 is modeled as a time series of five subsequent network cross-cuts.

\section{The Empirical Measurement of Domains' Symbiotic Interdependencies}

While the computation of domains' niche overlap is straightforward, two qualifications must be pointed out concerning the empirical measurement of domains' symbiotic interdependencies. First, the growth rate of each source domain $j$ is 
(marginally) affected by the citations $j$ receives from $i$. To eliminate this problem, we detracted all the citations $i$ makes to $j$, prior to calculating $y_{j t}$ in Equation 3 . Second, as we anticipated, a decision had to be made concerning which time lag should be used between $y_{j t}$ and $w_{i j t}$. That is, to measure the relative importance of each technology domain $j$ as a source of knowledge recombination for $i$ during $t$, when exactly should we calculate the proportion of patent citations $i$ makes to $j$ ? As an upper bound, one could choose to measure $y_{j t}$ and $w_{i j t}$ contemporaneously, i.e., within the same time interval. But to avoid potential problems of endogeneity, one could also argue that $w_{i j t}$ should be lagged with respect to $y_{j t}$ Because sorting out this issue on purely theoretical grounds appears impossible, we measured $w_{i j t}$ with six different lags, ranging from zero to five years before $y_{j t}$. We were glad to find that the results of our estimates of interest remained qualitatively unchanged. Indeed, both the effect size and the precision of our estimates improved with longer lags, which we interpret as a strong indication that the results do not reflect endogenous dynamics. In the statistical models reported here, $w_{i j t}$ was lagged by five years with respect to $y_{j t^{*}}$

\section{Analysis}

\section{Statistical Models}

To facilitate knowledge accumulation and guarantee comparability across studies, our sample and statistical models reproduce exactly Carnabuci and Bruggeman's (2009). Accordingly, we accounted for possible sources of unobserved heteoregeneity using both a fixed-effects and random-effects estimation (Hsiao 2003). We used a network disturbance model to get rid of possible interdependencies in the error (Leenders 2002). ${ }^{6}$ We included the full set of control variables used by Carnabuci and Bruggeman in their study. Namely, we included a set of time dummies that control for possible time-varying factors that could affect the growth of all technology domains in a similar way (e.g., macro-economic fluctuations, or changes in USPTO practices). We included a set of dummies to capture aggregate effects associated with the macro technological areas to which our technology domains belong (i.e., chemicals, mechanical, computer and communication, electric and electronic, pharmaceutical and drugs, and other). These dummies correspond to Hall et al.'s (2001) highest level classification of the USPTO patent classes, which has been developed by the authors as an integral part of the NBER Patent and Patent Citations Data Set. As Hall et al. (2001) showed, the validity of these six macro technological areas is reflected in a rich variety of patent and patent citations statistics, including aggregate tendencies to receive and make patent citations, the number of claims made per patent, and the degree of "generality" of the inventions patented. By now, Hall et al.'s classification is accepted as a standard proxy for broad technological areas in patent-based studies.

Because the vast majority of technological inventions are made by firms, we controlled for the (log of the) number of firms active in each technology domain 
during each time interval. It has been argued that the share of backward patent citations firms make to their own patents indicates their ability to appropriate the benefits of their own inventions, which may have an impact on firms' knowledgerelated investments and strategies (Hall et al. 2001). To ensure that these dynamics do not alter our estimates of interest, we computed for each technology domain during each time interval the average ratio of firm self-citations. Carnabuci and Bruggeman (2009) showed that technology domains tend to grow faster as they specialize in an increasingly homogeneous set of input ideas. However, the more specialized they become the slower their future growth rate; and the beneficial effect of increasing specialization decreases with the degree of specialization reached by a technology domain. In our study, we control for all three effects. As Carnabuci and Bruggeman point out, their measure of specialization does not take into account whether the degree of specialization of a technology domain results from a set of similarly specialized inventions or, rather, from a combination of highly specialized and highly brokering inventions. To account for these patent-level differences, the authors calculate for each technology domain during each time interval the coefficient of variation in patents' "originality," where the originality index is taken from Hall et al. (2001). This same variable is included as a control in our models. Lastly, we added a variable controlling for the total $\mathrm{R} \& \mathrm{D}$ dollars spent in each technology domain during each time interval. ${ }^{7}$ This control is important because technology domains are likely to grow faster if they experience more active government interventions or if they receive more $R \& D$ investments from firms. Because our control includes both private and public $R \& D$ investments, we account for these possible effects.

To make sure our statistical models are estimated on exactly the same sample as Carnabuci and Bruggeman, we removed the same 33 observations they removed from their study. Six of these are outliers. Twenty-three are technology domains that received no citations at all during a given time interval, which had to be removed because the concept of recombinant niche is meaningless in those cases. The last four dropped observations pertain to technology domain "miscellaneous." As a result of these choices, our sample comprises 1,639 observations out of 1,672, like Carnabuci and Bruggeman's.

\section{Results}

Before discussing the results of our econometric models, let us briefly look at the evolution of our explanatory and response variables. Interestingly, our data show that the extent of both competitive and symbiotic interdependencies among technology domains has grown substantially over the observation period, with an average yearly increase of roughly 1.5 percent and 3 percent, respectively. This generalized increase in ecological interdependencies seems consistent with the view, common among historians and theorists of technical change, that technological knowledge has become increasingly general and abstract over the years (Arora 


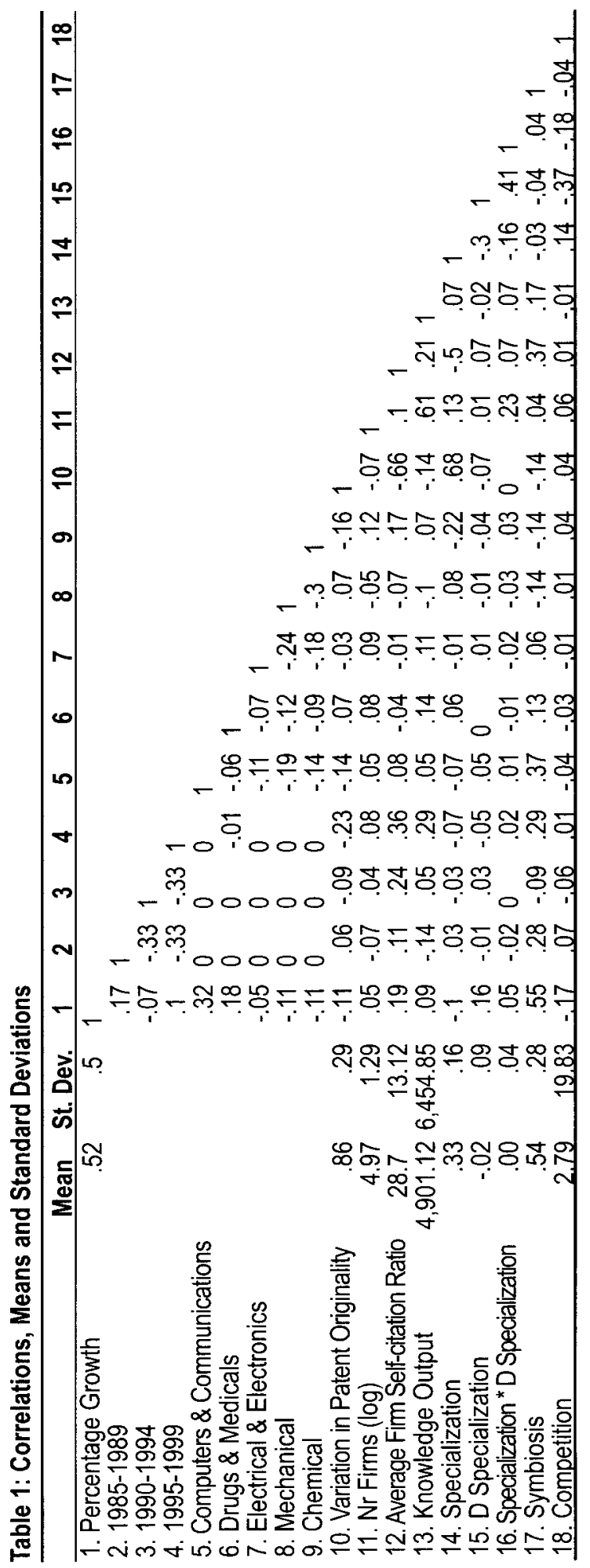


and Gambardella 1994), resulting in more pervasive knowledge recombinations across once far removed technology domains (Carnabuci, forthcoming). As the network of knowledge recombinations became more and more widespread, so did the ecological interdependencies that result from those recombinations.

When one breaks down the data by macro area of technology, however, it also becomes evident that two areas, "computer and communications" and "drugs and medicals," behaved somewhat differently from the rest of the technological landscape. In particular, these are the only two areas wherein competition has been consistently declining over the observation period, and where symbiotic interde-

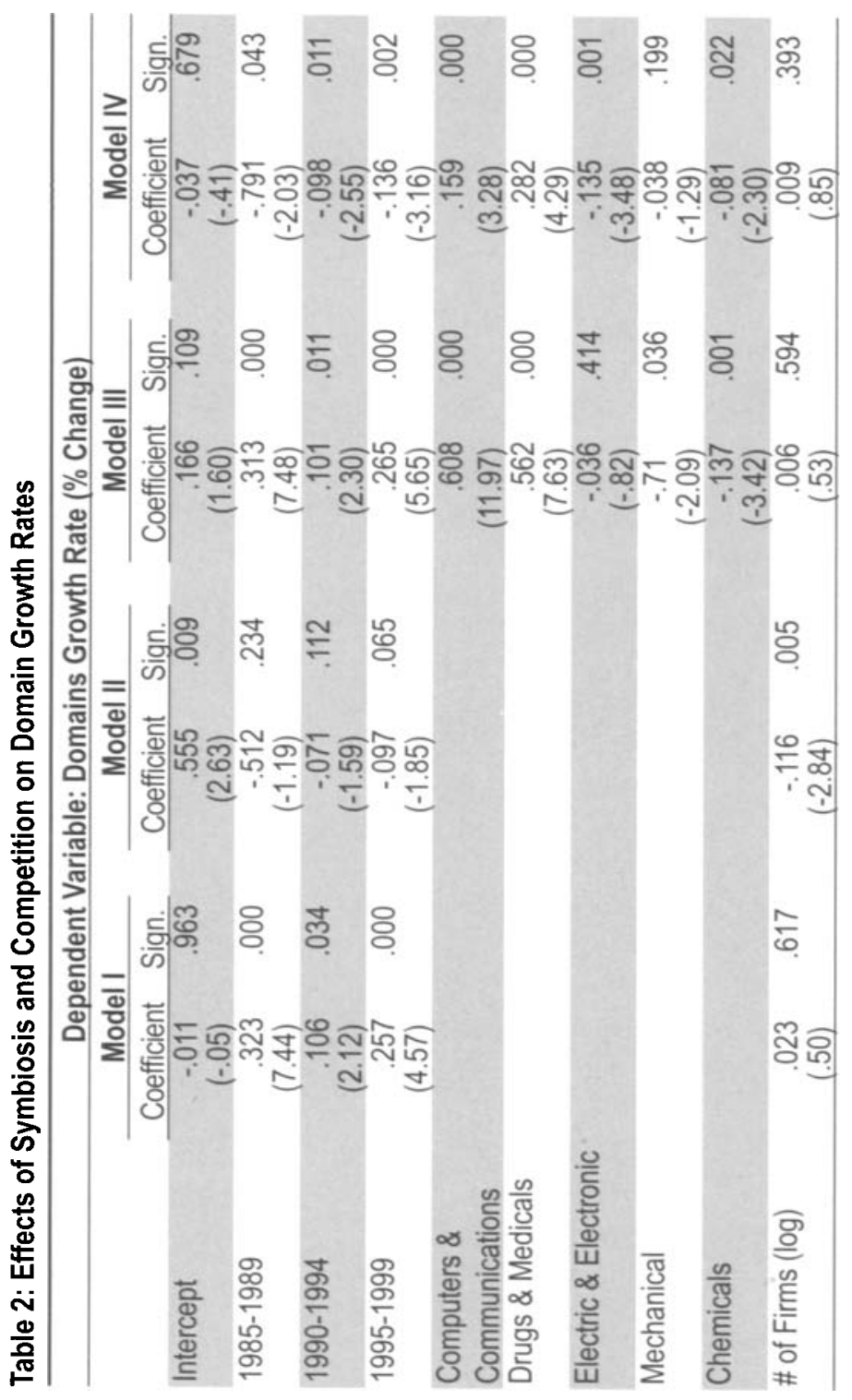


pendencies grew most rapidly. This is interesting because, as shown by Figure 5 and consistent with received wisdom, "computer and communications" and "drugs and medicals" underwent a spectacular progress during the observation period. These aggregate trends thus provide prima facie evidence in favour of our hypotheses.

Table 1 reports means, standard deviations, and correlations among the variables used in our statistical models. There appear to be no especially high correlations among the independent variables, as is confirmed by unproblematic multicollinearity statistics (the average VIF on the pooled data is 2.24). Notice that competition and symbiosis have a near-zero first-order correlation, suggest-

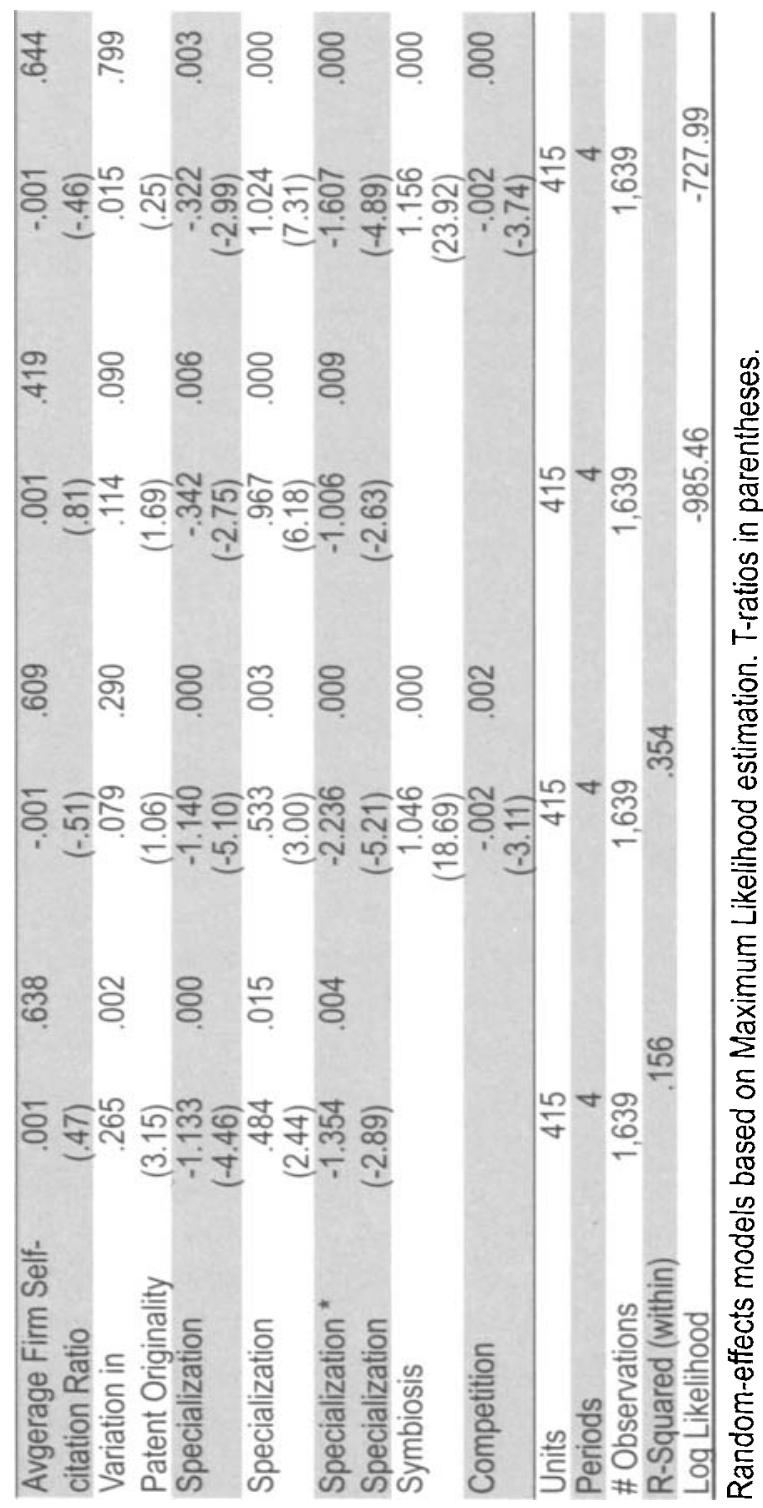


ing that the competitive pressure to which technology domains are subjected is independent of their symbiotic pressure, and vice-versa. Thus, a technology domain may at the same time be subjected to a high degree of competition and symbiosis or, by the same token, to a lack of both. This is in line with our theory and empirical constructs, which assume that symbiosis and competition are not endpoints of a conceptual continuum but, rather, orthogonal concepts capturing distinct ecological forces.

Table 2 displays the results of our statistical tests. Model 1 and Model 3 are baseline models based on fixed-effects and random-effects estimations, respectively. These models are identical to Carnabuci and Bruggeman's full models (2009). Model 2 and Model 4 add our two variables of interest (symbiosis and competition) to, respectively, Model 1 and Model 3. As said, our response variable is a domain's growth rate-domain's percentage growth between subsequent time intervals.

Consistent with received wisdom, models 1 and 3 show that the growth rates of technology domains tend to increase over time (albeit non-linearly). Indeed, economists have used this observation to substantiate their claim that unlike any other resource, the process of knowledge recombination yields increasing returns to scale (Mokyr 2002). When the effects of symbiosis and competition are controlled for, however, the trend changes quite dramatically. Based on analyses not reported in the paper, we observed that this change in the estimates of the time dummies is entirely due to the introduction in the model of the variable "symbiosis." We regard this finding as very interesting, as it suggests that the root cause of increasing returns to scale in the knowledge recombination process lies in the symbiotic interactions occurring among technology domains.

As we anticipated, models 3 and 4 indicate that the technological areas of "computers and communications" and "drugs and medicals" have been growing fastest. By contrast, the "chemical" sector has been growing significantly more slowly than the reference category "others" over the observation period. Although the results are less stark in the case of "electric and electronic" and "mechanical," also these sectors appear to have lagged behind. The number of firms technologically active in a domain has no effect on domains' growth rates, except in Model 2. Similarly, there seems to be at most a marginal effect associated with the copresence of highly specialized and highly "original" inventions in a technology domain. The three variables at the core of Carnabuci and Bruggeman's (2009) study, conversely, display the effects that were predicted by the authors. Namely, technology domains tend to grow significantly faster during periods in which their degree of specialization increases. However, the positive effect of increasing specialization is reversed for high levels of specialization and indeed, the higher the degree of specialization reached by a technology domain, the lower on average its future growth rate. Importantly, these results remain unchanged when the effects of symbiosis and competition among technology domains are controlled for, strengthening Carnabuci and Bruggeman's original test. 
Figure 3. Average Degree of Competitive Interdependence Among Technology Domains

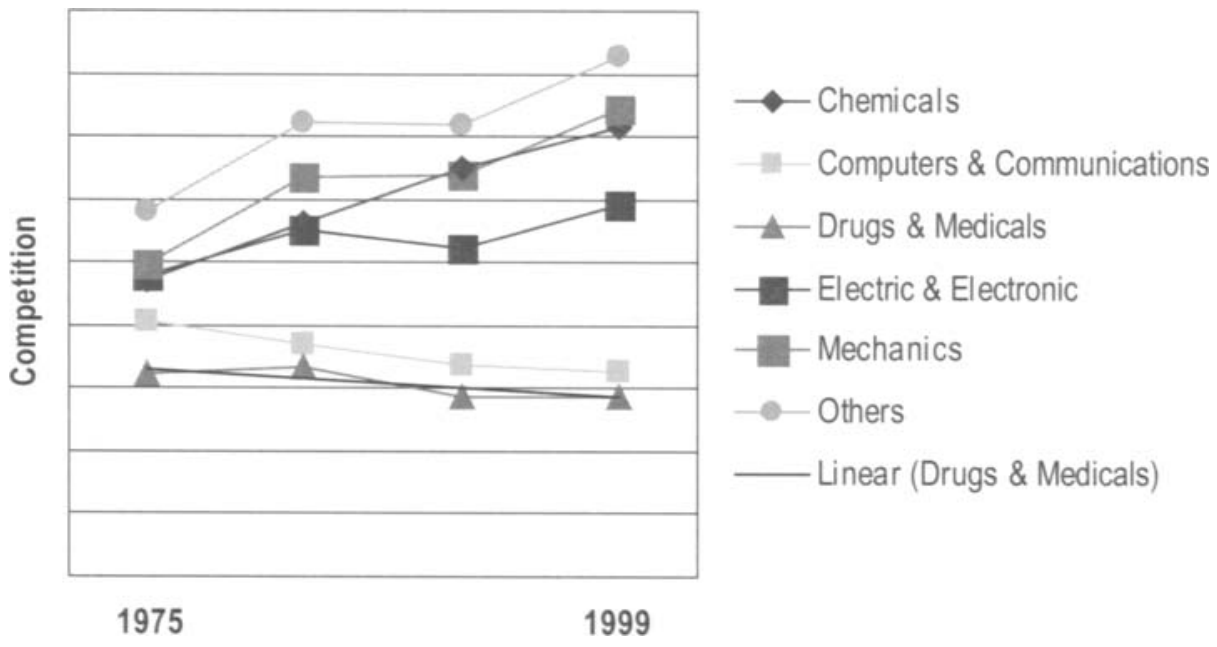

Figure 4. Average Degree of Symbiotic Interdependence Among Technology Domains

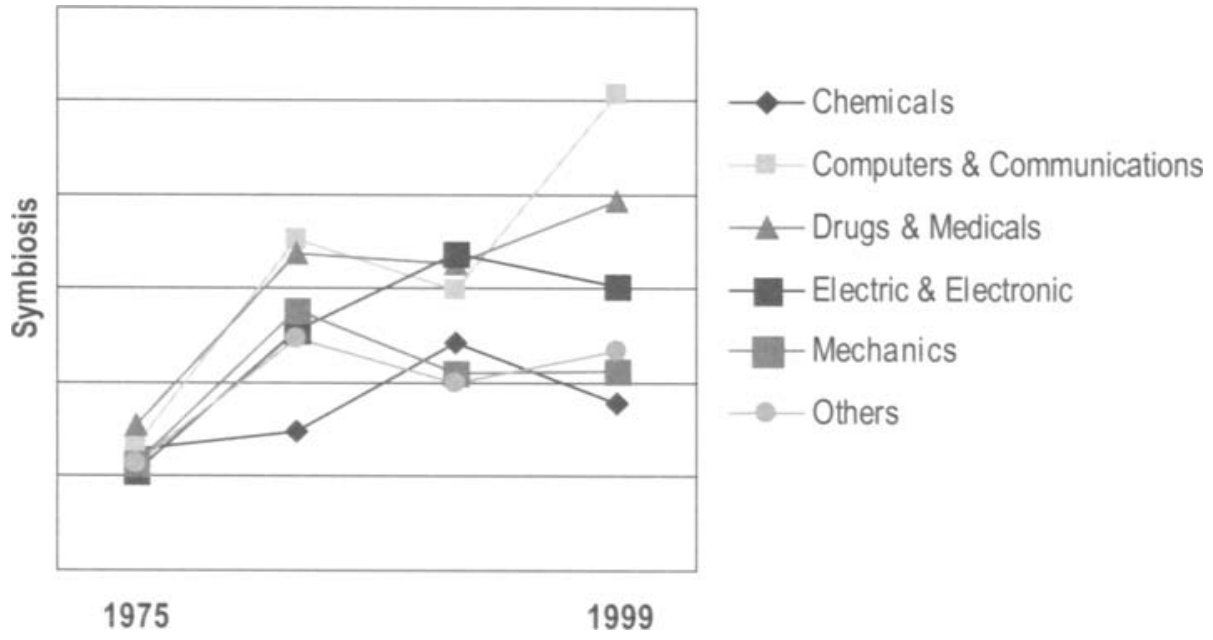

The last two rows of Model 2 and Model 4 report the estimates of our variables of interest. As predicted by Hypothesis 1, there exist symbiotic interdependencies among technology domains, so that the growth rate of any technology domain depends to a relevant extent on the growth rates of the technology domains it draws knowledge from. This effect is highly significant from a statistical point of view, with a t-ratio as high as 18.69 in the fixed-effects model and 23.92 in the random-effects one. In addition to a high degree of statistical significance, our estimates indicate that the role of symbiotic interdependencies among technology domains has a remarkable effect size. Based on our fixed-effects estimates, a one 
Figure 5. Average Growth Rate

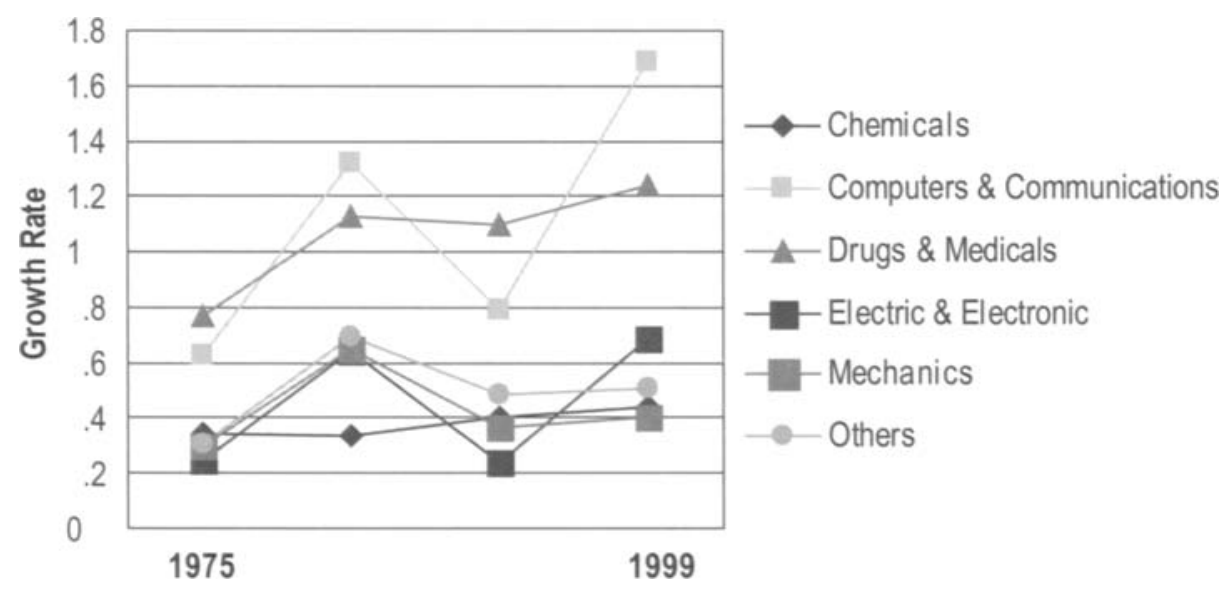

standard deviation difference in the amount of symbiotic pressure received by a focal technology domain can be expected to engender as much as a 29 percent increase in the domain's growth rate. This means that holding everything else constant, the growth rate of a technology domain embedded in high-growth position in the knowledge recombination network (top decile) is on average more than three times as large as the average growth rate of one embedded in a low-growth position (bottom decile).

Our Hypothesis 2 predicted that technology domains are in competition with each other to the extent that they tap knowledge inputs from overlapping recombinant niches. Based both on the fixed effects (Model 2) and the random effects (Model 4), the results of our analysis offer statistical support to this prediction. Hence, the more the overall niche overlap of a technology domain increases (decreases) over time, the more the domain's growth rate tends to decrease (increase). Namely, a one standard deviation increase in the overall niche overlap of a technology domain results on average in a 4 percent decrease in its growth rate, according to our fixed-effects estimate.

\section{Discussion and Conclusions}

In this article, we offered and documented a novel explanation for an important and widely acknowledged empirical phenomenon, i.e., that technology domains grow at different rates. Challenging the commonly held assumption that technology domains grow independently of one another, we argued and showed that technology domains are ecologically interdependent. We identified two kinds of ecological pressure that significantly impact the growth of technological knowledge across technology domains. First, we showed that because knowledge grows by recombination, and recombinant patterns cut across the boundaries of tech- 
nology domains, symbiotic interdependencies are pervasive among technology domains. Accordingly, the rate of growth of one technology domain tends to be accelerated by the advances made in other technology domains, to the extent that the latter is an habitual source of knowledge recombination for the former. Second, we showed that technology domains are in competition with each other insofar as they tap knowledge from overlapping recombinant niches. As a result, the rate at which a given technology domain advances varies inversely with the competitive pressure it receives from overlapping technology domains. Due to competition and mutualism, and unlike conventional accounts of technological progress, this article showed that technological progress is an inherently ecological process wherein the growth rate of each technology domain depends on dynamics occurring in other technology domains.

We think that the article makes four main contributions. First, it extends the line of inquiry initiated by Carnabuci and Bruggeman's study of the growth of technology domains (2009). Employing a characteristically sociological toolkit to analyze the growth of public technological knowledge, Carnabuci and Bruggeman proposed that the process of technological knowledge growth is best conceived as an evolving network of knowledge recombination patterns among technology domains. Through this perspective, the authors were able to show that the growth of technology domains depends on intertwined dynamics of knowledge specialization and knowledge brokerage. In the present article, we built on Carnabuci and Bruggeman's intuition and model to study how technology domains "grow together" (Girifalco 1991), thereby offering a distinctly ecological interpretation of their network conception of technological progress. As we are confident that the space of research opportunities opened up by the network perspective on technological progress is still ample, we regard the possibility of further knowledge accumulation along this line of inquiry as both promising and desirable. Indeed, both the mathematical formalization provided by network methods and the availability of the NBER public data set ought to render the pursuit of knowledge accumulation easier in this case than in many other sociological researches.

Second, thus far most scholars have been persuaded that because knowledge is a non-rival resource, the knowledge recombination process is unboundedly selfreinforcing; hence, the rate of knowledge generation is increasing in scale. In a pair of influential articles, for example, Weitzman $(1996,1998)$ argued that given sufficient $\mathrm{R} \& \mathrm{D}$ investments, the stock of technological knowledge will increase combinatorially. We qualified Weitzman's work by explicating a mechanism-competition among technology domains - that acts as an endogenous constraint in the knowledge recombination process. Indeed, Weitzman (1998:354, italics added) alluded to the possible existence of competition in knowledge recombination by remarking that "[i]f there were a finite number of potential ideas, then one researcher might create negative externalities for other researchers through a process akin to overfishing in a crowded pond." Our study substantiated this speculative remark, showing 
that the negative externalities of competition are an inherent and non-negligible characteristic of the knowledge recombination process. Besides being an important theoretical qualification per se, accounting for the role of knowledge competition suggests that the productivity of knowledge recombination may decline even in the presence of increasing $R \& D$ resources - a conclusion that helps to make sense of "... the well-known 'puzzle' of the large fall in the ratio of US patents to US research inputs in the post-war period."(Caballero and Jaffe 1993:50)

To be sure, we agree with Weitzman and the proponents of so-called New Growth Theory (Jones 2005) that the process of knowledge recombination can and often does generate symbiotic externalities and increasing returns to scale. However, rather than assuming a priori an unconstrained combinatorial knowledge growth across the board, our proposed ecological perspective indicates that the extent of symbiotic pressure varies among technology domains depending on their position in the knowledge recombination network. In this respect, a third contribution of our study is that it provides a useful middle point between two well-established, yet starkly contrasting, visions of technological progress. On the one hand, as noted by Harberger (1998), economists have proposed a "yeast" vision of technological progress, wherein the positive externalities of technological advances are implicitly assumed to spread out homogeneously throughout the whole technological landscape. Indeed, the very concept of technology domain is irrelevant according to this vision, and technological progress may be aptly modelled à la Weitzman. On the other hand, the evolutionary view of technological change (Dosi 1982, Nelson and Winter 1982) has maintained that each technological domain has highly path-dependent and idiosyncratic dynamics, implying that the growth trajectories of technology domains are largely independent of each other. Our ecological perspective indicates that technological knowledge grows neither as "yeast," nor as a collection of independent technology domains. Rather, the growth of technology domains is driven by an evolving network of symbiotic interdependencies whose structure is determined by the actual pattern of knowledge recombination taking place among them.

A fourth contribution of this article is that it offers a novel perspective on the role of technology policy. ${ }^{8}$ Reflecting the traditional view that technology domains grow independent of one another, scholars have noted that in order to favour the development of specific technology domains, it is imperative to increase the $R \& D$ investments therein. Implied from this argument is the precept that policy makers should aim to counteract "market failures" by funnelling R\&D resources towards socially desirables technologies, either directly through public R\&D spending or by providing a suitable incentive structure to private investors (Tassey 1997). A notable example occurred in the nineties, when the U.S. government proposed a multi-billion budget for government-funded $R \& D$ and tax credits to foster the progress of renewable-energy technologies. While it is obvious that $R \& D$ spending influences the rate and direction of technological progress, our analyses 
showed that the ecological dynamics of competition and symbiosis exert a strong additional effect. Rather than merely increasing the level of $R \& D$ investment on a given technology domain, we therefore suggest that policy makers should use their resources to take better advantage of the growth dynamics occurring in other technology domains. Namely, our results indicate that to boost the progress of a given technology domain, $R \& D$ resources should be allocated in such a way that the competitive interdependencies to which it is subjected are minimized, while its symbiotic ones are maximized. For example, government interventions may favour the cross-domain projects that involve fast-growing technology domains, or that reduce dependence on sluggish and crowded ones.

In concluding, we would like to acknowledge that our study suffers from at least two noteworthy limitations. First, our analyses are based exclusively on patent-based indicators. Whether and to what extent competitive and symbiotic interactions occur in non-patented technological knowledge is a question that ought to be addressed. Second, we took a markedly macroscopic perspective. By so doing, we abstracted away from knowledge growth mechanisms taking place at lower levels of analysis, particularly the business firm. To be sure, extant research indicates that the ecological dynamics of knowledge competition (Podolny and Stuart 1995) and symbiosis (Operti and Carnabuci 2008) do affect firms' ability to generate new technologies. However, we do not yet know how these ecological dynamics affect the growth rate of technology domains. For example, there is evidence showing that the more a firm competes for knowledge with other firms, the less it tends to grow (Podolny et al. 1996); but how the growth rate of technology domains is affected by different degrees of inter-firm knowledge competition is an open question and, we believe, a potentially fruitful avenue of future research.

\section{Notes}

1. The present article extends Carnabuci and Bruggeman's (2009) networkbased conception of technological progress by examining the role of ecological interdependencies among technology domains. Unlike previous analytical approaches, a distinguishing aspect of Carnabuci and Bruggeman's network model of technological progress is that it assumes that technology domains are dependent on each other as sources of knowledge recombination. In their article, however, Carnabuci and Bruggeman did not explore, either theoretically or empirically, the ecological implications of this assumption. Rather, they examined how the growth of technology domains varies as they become more or less specialized. By contrast, we build on Carnabuci and Bruggeman's intuition and network model to derive and test an ecological theory of technological progress. Namely, we argue that net of the specialization effects found by Carnabuci and Bruggeman and other relevant domain-specific factors, the growth of each technology domain depends on dynamics occurring in other technology domains.

2. Symbiotic interdependence is here defined as a relationship between two entities, in our case technology domains, wherein at least one entity derives some kind of fit benefit from the other, while the fit of the latter is not reduced. Two aspects of this definition deserve to be mentioned. First, symbiotic interdependencies can, but do not need to 
be, symmetric. Thus, the degree of symbiotic dependence of technology domain $i$ on technology domain $j$ may be different from the degree of symbiotic dependence from $j$ to $i$. And indeed, $i$ may be symbiotically dependent on $j$ even if $j$ is not symbiotically dependent on $i$. Notice that this definition is consistent with the etymology of the compound term interdependence (i.e., dependence between), which denotes a dependence relationship between distinct entities, but not necessarily a symmetric one. Second, our definition of symbiotic interdependence excludes predatory and parasitic relations, wherein the fit benefit accruing to one entity corresponds to a fit deficit in the other (e.g., the fox benefits from eating hares, but this reduces the fit of hares).

3. For the sake of simplicity, in this example we assumed that the knowledge recombination patterns on which $w_{i j t}$ is calculated are contemporaneous to $y_{j t}$ (i.e., we assumed that there should be no time lag between $w_{i j t}$ and $y_{j t}$ ). As we will discuss later in the article, however, this choice is not obvious, as one might argue that $w_{\text {iit }}$ should be lagged with respect to $y_{i{ }^{\circ}}$ Because the choice of any specific time lag would be somewhat arbitrary, for the empirical test of our argument we will use several operationalizations of $w_{i j t}$, with time lags ranging from zero to five years.

4. For an illustration of asymmetry, the niche overlap of $i$ on $k$ in Figure 1 is $\alpha_{k i, t}=(20$ $+50) / 185=.378$. As can be seen, because the total number of recombinations by $k$ is smaller than $i$ s, the overlap of $i$ on $k$ is larger than the overlap of $k$ on $i$.

5. Alcácer and Gittelman (2006) have shown that because many patent citations are inserted by the USPTO patent examiners after the patent has been applied for, if one uses patent citations to infer which prior inventions an inventor consciously built on to generate his/her own invention, one is likely to incur both type I and type II errors. However if one is interested, as we are, in mapping how knowledge is recombined across the technological landscape, the additional citations inserted by patent examiners provide a valuable integration (Sorenson and Singh 2007).

6. Network disturbance models use information on the network structure of the data to account for a possible lack of independence in the error terms of the regression equation. This kind of models assumes that there are patches of interrelated cases that fit the prediction of the statistical model better that one would expect by chance, patches for which the model predictions are systematically underestimated and, finally, patches for which the model predictions are systematically overestimated (Dow et al. 1984). Let $y$ be a $(n \times 1)$ vector of values of a network autocorrelated variable for the $n$ nodes of a network, let $X$ denote a $(n \times k)$ matrix of values for the $n$ nodes on $k$ covariates and let $W$ be a $(n \times n)$ "weight" matrix based on the network data. Following Carnabuci and Bruggeman (2009), we modelled the weight matrix $W$ by row-normalizing our domain-to-domain patent citation adjacency matrix. Within this framework, a network disturbance model accounts for the effects of network autocorrelation by modeling parameter $\rho$ in the following statistical equation:

$$
y=X \beta+\varepsilon, \quad \varepsilon=\rho W \varepsilon+\nu, \quad \nu \sim N(0, \sigma 2 I)
$$

7. Although publicly available, these $R \& D$ data contain many missing values. For that reason, Carnabuci and Bruggeman (2009) ran a separate batch of models with this additional control, which they did not report in the paper. We did the same. Notwithstanding the great reduction in sample size, our results remained qualitatively unchanged with regard to our variables of interest, when the R\&D control was included in the model.

8. We are grateful to an anonymous reviewer for pointing out that our theory could be related to technology policy making. 


\section{References}

Albert, M.B., D. Avery, F. Narin and P. McAllister. 1991. "Direct Validation of Citation Counts as Indicators of Industrially Important Patents." Research Policy 20(3):251-59. Alcácer, Juan, and Michelle Gittelman. 2006. "How Do You Know What You Know? The Role of Inventors and Examiners in the Generation of Patent Citations." Review of Economics and Statistics 88(4):774-79.

Arora, Ashish, and Alfonso Gambardella. 1994. "The Changing Technology of Technological Change: General and Abstract knowledge and the Division of Innovative Labour." Research Policy 23(5):523-32.

Arrow, Kenneth J., 1962. "Economic Welfare and the Allocation of Resources for Inventions." Pp. 609-26. The Rate and Direction of Inventive Activity: Economic and Social Factors. R.R. Nelson, editor. Princeton University Press.

Barnett, William P., and Glenn R. Carroll. 1987. "Competition and Mutualism among Early Telephone Companies." Administrative Science Quarterly 32(3):400-21.

Bonaccorsi, Andrea, and Grid Thoma. 2007. "Institutional Complementarity and Inventive Performance in Nano Science and Technology." Research Policy 36(6):813-31.

Brittain, Jack, and Douglas H. Wholey. 1988. "Competition and Coexistence in Organizational Communities: Population Dynamics in Electronics Components Manufacturing." Pp. 195-222. Ecological Models of Organizations. G.R. Carroll, editor. Ballinger.

Bruggeman, Jeroen, Gianluca Carnabuci and Ivar Vermeulen. 2003. "A Note on Structural Holes Theory and Niche Overlap." Social Networks 25(1):97-101.

Caballero, Ricardo J., and Adam B. Jaffe. 1993. How High are the Giants'Shoulders: An Empirical Assessment of Knowledge Spillovers and Creative Destruction in a Model of Economic Growth. NBER Macroeconomics Annual, Volume 8. O. Blanchard and S. Fischer, editors. MIT Press.

Carnabuci, Gianluca. 2006. A Theory of Knowledge Growth. Amsterdam University Press. . Forthcoming. "The Evolving Structure of the Technological Landscape." Technology Analysis and Strategic Management.

Carnabuci, Gianluca, and Jeroen P. Bruggeman. 2009. "Knowledge Specialization, Knowledge Brokerage, and the Uneven Growth of Technology Domains." Social Forces 88(2):607-41.

Doreian, Patrick, Klaus Teuter and Chi-Hsein Wang. 1984. "Network Autocorrelation Models: Some Monte Carlo Evidence." Sociological Methods and Research 13(2):155-20.

Dosi, Giovanni. 1982. "Technological Paradigms and Technological Trajectories." Research Policy 11(3):47-62.

Dow, Malcom M., Michael L. Burton and Douglas R. White. 1982. "Network Autocorrelation: A Simulation Study of a Foundational Problem in Regression and Surveyresearch." Social Networks 4(2):169-200.

Durkheim, Émile. 1997[1893]. The Division of Labor in Society. The Free Press.

Fleming, Lee, Santiago Mingo and David Chen. 2007. "Collaborative Brokerage, Generative

Creativity, and Creative Success." Administrative Science Quarterly 52(3): 443-75.

Girifalco, Louis A. 1991. Dynamics of Technological Change. Van Nostrand Reinhold.

Griliches, Zvi. 1979. "Issues in Assessing the Contribution of Research and Development to Productivity Growth." Bell Journal of Economics 10(1):92-116. . 1990. "Patent Statistics as Economic Indicators: A Survey." Journal of Economic Literature 28(4):1661-707. 
Gross, Edward. 1956. "Symbiosis and Consensus as Integrative Factors in Small Groups." American Sociological Review 21(2):174-79.

Hall, Bronwyn, Adam B. Jaffe and Manuel Trajtenberg. 2001. The NBER Patent Citations Data File: Lessons, Insights and Methodological Tools. Available at: http://www.nber.org/patents.

Harberger, Arnold C. 1998. "A Vision of the Growth Process." American Economic Review $88(1): 1-32$.

Hawley, Amos H. 1950. Human Ecology: A Theory of Community Structure. The Ronald Press Company.

Henderson, Rebecca, Adam B. Jaffe and Manuel Trajtenberg . 2005. "Patent Citations and the Geography of Knowledge Spillovers: A Reassessment: Comment." American Economic Review 95(1):450-60.

Hsiao, Cheng, 2003. Analysis of Panel Data. 2nd Edition, Cambridge University Press.

Ingram, Paul, and Tal Simons 2000. "State Formation, Ideological Competition, and the Ecology of Israeli Workers Cooperatives, 1920-1992." Administrative Science Quarterly 45(1):25-53.

Jaffe, Adam B. 1986. "Technological Opportunity and Spillovers of R\&D: Evidence from Firms' Patents, Profits and Market Value." American Economic Review 76(5):984-1001. Jaffe, Adam B., Michael S. Fogarty and Bruce A. Banks. 1988. "Evidence from Patents and Patent Citations on the Impact of NASA and other Federal Labs on Commercial Innovation." Journal of Industrial Economics 46(2):183-205.

Jaffe, Adam B., Manuel Trajtenberg and Michael S. Fogarty. 2000. "Knowledge Spillovers and Patent Citations: Evidence from a Survey of Inventors." American Economic Review 90(2):215-18.

Jaffe, Adam B., Manuel Trajtenberg and Rebecca Henderson. 1993. "Geographic Localization of Knowledge Spillovers as Evidenced by Patent Citations." Quarterly Journal of Economics 108(3):577-98.

Jones, Charles. 2005. "Growth and Ideas." Pp. 1064-108. Handbook of Economic Growth. P. Aghion and S. Durlauf, editors. North-Holland.

Knorr-Cetina, Karin D. 1999. Epistemic Cultures. How the Sciences Make Knowledge. Harvard University Press.

Latour, Bruno 1987. Science in Action: How to Follow Scientists and Engineers Through Society. Harvard University Press.

Leenders, Roger T.A.J. 2002. "Modeling Social Influence through Network Autocorrelation: Constructing the Weight Matrix." Social Networks 24(1):21-47

Malerba Franco, Richard R. Nelson, Luigi Orsenigo and Sidney Winter. 1999. "History Friendly Models of Industry Evolution: The Case of the Computer Industry." Industrial and Corporate Change 8(1):3-40.

Mokyr, Joel, 2002. The Gifts of Athena: Historical Origins of the Knowledge Economy. Princeton University Press.

Nelson, Richard R., 2003. "On the Uneven Evolution of Human Know-How." Research Policy 32(6):909-22.

Nelson, Richard R., and Sidney Winter. 1982. An Evolutionary Theory of Economic Change. Harvard University Press.

Operti, Elisa, and Gianluca Carnabuci. 2008. "Knowledge that Flows and Knowledge that Grows: Inventive Performance in the Semiconductor Field." Best Paper Proceedings of the Academy of Management. Available at: www.aomonline.org. 
Park, Robert. 1936. "Human Ecology." American Journal of Sociology 42(1):1-15.

Pianka, Eric R. 1978 Evolutionary Ecology, 2nd Edition. Harper \& Row.

Pistorius, Carl W.I., and James M. Utterback. 1997. "Multi-Mode Interaction among Technologies." Research Policy 26(1):67-84.

Podolny, Joel M., and Toby E. Stuart. 1995. "A Role-Based Ecology of Technological Change." American Journal of Sociology 100(5):1224-6.

Podolny, Joel M., Toby E. Stuart and Michael T. Hannan. 1996. "Networks, Knowledge, and Niches: Competition in the Worldwide Semiconductor Industry, 1984-1991." American Journal of Sociology 102(3):659-89.

Popielarz, Pamela A., and Zachary P. Neal. 2007. "The Niche as a Theoretical Tool." Annual Review of Sociolology 33:65-84.

Rogers, Everett M. 2003. The Diffusion of Innovations, $5^{\text {th }}$ Edition. Free Press.

Romer, Paul M. 1986. "Increasing Returns and Long-Run Growth." Journal of Political Economy 94(5):1002-37.

Schmookler, Jacob. 1966. Invention and Economic Growth. Harvard University Press.

Sohn, M.W. 2001. "Distance and Cosine Measures of Niche Overlap." Social Networks 23(2):141-65.

Sorenson Olav, and Jasjit Singh. 2007. "Science, Social Networks and Spillovers." Industry and Innovation 14(2):219-38.

Tassey, Gregory, 1997. The Economics of R\&D Policy. Quorum Books.

Usher, Abbort P. 1929. A History of Mechanical Inventions. McGraw-Hill.

Weitzman, Martin L. 1996. "Hybridizing Growth Theory." American Economic Review $86(2): 207-12$.

. 1998. "Recombinant Growth." Quarterly Journal of Economics 86(2):331-60. 